\title{
INTERRELATIONS OF METEORITES, ASTEROIDS, AND COMETS
}

\author{
EDWARD ANDERS \\ University of Chicago
}

\begin{abstract}
Most meteorites come from a small number of parent bodies (6 to 11), with radii mainly between 100 and $300 \mathrm{~km}$. The most likely sources of meteorites are seven asteroid families between 1.9 and $2.8 \mathrm{AU}$, whose eccentricities and inclinations are high enough to permit their collisional debris to cross the orbit of Mars. Comets are too small and too numerous to serve as a source of the major meteorite classes, but they may well be an important source of micrometeorites and carbonaceous chondrites.
\end{abstract}

Asteroids and comets are the two most plausible sources of meteorites. However, there is as yet no agreement on the relative importance of the two. Some authors believe that the great majority of meteorites come from comets (Öpik, 1965, 1968; Wetherill, 1968a). Others argue that they come mainly from asteroids (Anders, 1964; Wood, 1968). Let us review the principal clues to the nature of meteorite parent bodies, as obtained from the meteorites themselves.

\section{NUMBER AND SIZE OF METEORITE PARENT BODIES}

\section{Irons}

Wasson has shown in an impressive series of papers (Wasson, 1969, 1970, and earlier publications cited therein) that most iron meteorites fall into 11 discrete groups, differing from each other in chemical composition and structure. In four-dimensional composition space ( $\mathrm{Ga}, \mathrm{Ge}, \mathrm{Ir}$, and $\mathrm{Ni}$ ) these groups form very compact, well-defined clusters. Many of these clusters can also be recognized by other criteria, such as radiation age and shock effects (Jaeger and Lipschutz, 1967; Voshage, 1967). The observed infall rate of these meteorites requires a source at least 1 to $100 \mathrm{~km}^{3}$ in extent; and it therefore seems highly probable that each group represents either a separate parent body, or a sizable, compositionally distinct region within a parent body.

Clues to the size of these bodies have been obtained from the Widmanstätten pattern of iron meteorites. The formation of this pattern, long a subject of controversy, is now well understood, thanks to the work of Wood 
(1964), Goldstein and Doan (1971), and Goldstein and Ogilvie (1965). As a result of this understanding, it has been possible to estimate cooling rates of iron meteorites through the range in which the pattern formed, 700 to $300 \mathrm{C}$. The results for nearly 300 iron meteorites and pallasites range from 0.4 to $500 \mathrm{~K} /$ million $\mathrm{yr}$, with the majority of values lying between 1 and $10 \mathrm{~K} /$ million yr (table I).

These metallographically determined cooling rates are a direct clue to the size of the parent bodies, because the cooling rate of a planetary object is a sensitive function of size. Fricker et al. (1970) have shown that the above cooling rates correspond to radii between 10 and $500 \mathrm{~km}$, with most values lying between 100 and $300 \mathrm{~km}$. Some of Wasson's groups show little spread in cooling rates, which suggests that they come from a (nearly isothermal) core. Others show a nearly tenfold variation, which may imply that they are derived from a series of isolated iron pools extending from the center to the surface (Urey, 1966). At cooling rates less than $7 \mathrm{~K} /$ million yr, the radius depends strongly on whether the outer layers of the body are compositionally uniform or differentiated, with radioactive elements concentrated near the surface. Two different radii are therefore given in table I, for the uniform and differentiated cases.

The cooling rates have been confirmed by an independent method: fissiontrack measurements in the Toluca iron meteorite (Fleischer et al., 1968). By measuring tracks from extinct, 82 million yr ${ }^{244} \mathrm{Pu}$ in three minerals differing in track retention temperatures, Fleischer et al. obtained three points on a cooling curve for Toluca. The cooling rate found, $1.1_{-0.5}^{+0.6} \mathrm{~K} /$ million yr, agrees well with the metallographically determined value, $1.6 \pm 0.6 \mathrm{~K} / \mathrm{million} \mathrm{yr}$

TABLE I.-Parent Bodies of Iron Meteorites

\begin{tabular}{|c|c|c|c|c|c|}
\hline \multirow{2}{*}{$\begin{array}{l}\text { Chemical } \\
\text { group }\end{array}$} & \multirow{2}{*}{$\begin{array}{c}\text { Cooling } \\
\text { rate, } \\
\mathbf{K} / \text { million } y r\end{array}$} & \multirow{2}{*}{$\begin{array}{l}\text { Number } \\
\text { of } \\
\text { members }\end{array}$} & \multicolumn{2}{|c|}{ Radius, $\mathrm{km}^{\mathrm{c}}$} & \multirow{2}{*}{$\begin{array}{l}\text { Radiation } \\
\text { age, d } \\
\text { million yr }\end{array}$} \\
\hline & & & $\begin{array}{c}\text { Uniform } \\
\text { body }\end{array}$ & $\begin{array}{c}\text { Differentiated } \\
\text { bcdy }\end{array}$ & \\
\hline$I \ldots \ldots \ldots$ & 1 to 3.5 & 87 & $150 \pm 30$ & $230 \pm 70$ & 900 \\
\hline IIA $\ldots .$. & $\ldots \ldots$ & 29 & 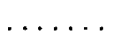 & $\cdots$ & $\leqslant 100$ \\
\hline IIB & 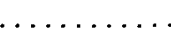 & 11 & $\cdots$ & $\ldots \ldots$ & 1000 \\
\hline IIC & 100 to 250 & 7 & $25 \pm 5$ & $25 \pm 5$ & $\ldots \ldots$ \\
\hline IID $\ldots$ & 1 to 2 & 6 & $165 \pm 15$ & $260 \pm 40$ & $\sim 1000$ \\
\hline IIIA $\ldots$ & 1.5 to 10 & 24 & 170 & 250 & 700 \\
\hline IIIB $\ldots$ & 1 to 2 & 14 & $165 \pm 15$ & $260 \pm 40$ & 700 \\
\hline IIIC & 2 to 5 & 5 & $130 \pm 10$ & $180 \pm 40$ & 700 \\
\hline IIID $\ldots \ldots$ & 2 to 5 & 5 & $130 \pm 20$ & $180 \pm 40$ & 200 \\
\hline IVA $\ldots \ldots$ & 7 to 90 & 16 & 100 & 110 & 400 \\
\hline IVB & 2 to 20 & 7 & 150 & 220 & Variable \\
\hline
\end{tabular}

aWasson $(1969,1970$, and earlier publications cited therein.)

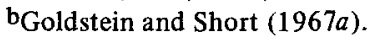

cFricker et al. (1970).

dVoshage (1967). 
(Goldstein and Short, 1967b). Although a few uncertainties remain, the metallographic cooling rates for iron meteorites are probably reliable to within a factor of 2 to 3 .

The actual number of parent bodies involved may be as small as six. Though the subgroups IIIA to IIID are chemically distinct from each other, the differences are not drastic and in fact were not noticed until very precise analyses became available. Perhaps all came from the same body. All give essentially the same radius $r$; at least three of the four were produced in a single collision, judging from their common radiation age of $\sim 700$ million $\mathrm{yr}$ and the ubiquity of strong shock effects (Jaeger and Lipschutz, 1967). Similarly, IIA, B, and D may come from a single body. Assuming independent bodies for I, IIC, IVA, and IVB, we are thus left with only six bodies. Apparently more than 80 percent of all iron meteorites came from 11 bodies at most (possibly as few as six), of which all but 1 were larger than $100 \mathrm{~km}$ in radius.

\section{Chondrites}

According to chemical criteria, chondrites are divided into five groups (table II). The hiatuses separating these groups are not as wide as those for irons, and hence there is less reason to conclude, on chemical grounds, that only five parent bodies are involved. Here we must rely on other evidence.

Among the meteorites in table II, the L-chondrites stand out in having a preponderance of short $\mathrm{K}$-Ar and U-He ages, discordant between 1 and 4 aeons, but becoming concordant at $\sim 0.5$ aeon. These short ages are correlated with shock and reheating symptoms. Detailed analysis of the data suggests that at least two-thirds of these meteorites were involved in a major collision $520 \pm 60$ million yr ago, which caused partial or complete outgassing of ${ }^{40} \mathrm{Ar}$ and ${ }^{4} \mathrm{He}$ (Anders, 1964; Heymann, 1967; Taylor and Heymann, 1969).

\section{TABLE II.-Classification of Chondrites}

[Van Schmus, 1969]

\begin{tabular}{|c|c|c|c|c|c|}
\hline Chemical group & $\frac{F e_{\text {metal }}}{F e_{\text {total }}}$ & $\mathrm{Fe} / \mathrm{Si}$ & $\mathrm{Mg} / \mathrm{Si}$ & $\frac{\mathrm{Fe}^{2+}}{\mathrm{Fe}^{2+}+\mathrm{Mg}^{2+}}$, & $\begin{array}{c}\text { Known } \\
\text { falls }\end{array}$ \\
\hline $\left.\begin{array}{l}\text { E, enstatite } \\
\mathrm{H} \\
\mathrm{L} \\
\mathrm{LL}\end{array}\right\}$ ordinary ${ }^{\mathrm{b}}$ & $\begin{array}{l}0.80 \pm 0.10 \\
.63 \pm .07 \\
.33 \pm .07 \\
.08 \pm .07 \\
\text { Low }\end{array}$ & $\begin{array}{c}0.83 \pm 0.32 \\
.83 \pm .08 \\
.59 \pm .05 \\
.53 \pm .03 \\
.83 \pm .08\end{array}$ & $\begin{array}{c}0.79 \pm 0.06 \\
.96 \pm .03 \\
.94 \pm .03 \\
.94 \pm .03 \\
1.05 \pm .03\end{array}$ & $\begin{array}{l}0 \text { to } 2 \\
16 \text { to } 20 \\
22 \text { to } 26 \\
27 \text { to } 31 \\
33\end{array}$ & $\begin{array}{r}11 \\
225 \\
273 \\
39 \\
36\end{array}$ \\
\hline
\end{tabular}

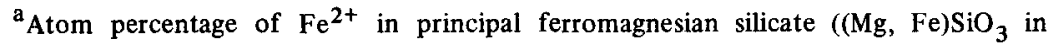
enstatite chondrites and $(\mathrm{Mg}, \mathrm{Fe})_{2} \mathrm{SiO}_{4}$ in all others).

${ }^{b}$ The symbols H, L, and LL refer to total iron content, and stand for high, low, and low-low, respectively. 
If this is true, then the L-chondrites come mainly from one or at most two bodies. Because L-chondrites are the most abundant class of meteorites now falling on Earth, it seems that the majority of chondrites, like the irons, come from a small number of bodies: probably no more than 10 , perhaps as few as five. The total number of bodies need not be greater than perhaps six because some chondrites and irons may come from the same body. For example, the outgassing time of L-chondrites, $520 \pm 60$ million $\mathrm{yr}$, is rather close to the radiation age of group III irons, 600 to 700 million yr (Vosháge, 1967), and the systematic errors in the two dating methods are large enough to admit the possibility that both refer to the same event. Chemical resemblances between these two classes are sufficiently great to permit an origin in the same body.

Wänke (1966) and Öpik (1968) have questioned the reality of the 520 million yr event, and the conclusion that most L-chondrites come from a single parent body. They suggest that the meteorite parent body was hot enough throughout its history to cause ${ }^{4} \mathrm{He}$ to partition between solid and pore space. On breakup, the ${ }^{4} \mathrm{He}$ in the pores would escape, leaving only a fraction of the total ${ }^{4} \mathrm{He}$ in the meteorite. If this fraction happened to be 8 percent, an apparent $\mathrm{U}-\mathrm{He}$ age of 520 million yr would result.

However, this explanation appears to be untenable. It does not account for the fact that U-He ages of 0.5 aeon are sometimes associated with concordant $\mathrm{K}$-Ar ages of 0.5 aeon, and sometimes with discordant values as high as 3 aeons. It predicts a correlation between U-He age and porosity that is not observed, and yet fails to explain the correlation between U-He or K-Ar age and shock effects that is observed. (This correlation has been confirmed by several authors: Carter et al., 1968; Christophe, 1969; Taylor and Heymann, 1969; Van Schmus and Ribbe, 1968; Wood, 1967.) Finally, the reality of the 520 million $\mathrm{yr}$ outgassing event has been confirmed by the ${ }^{40} \mathrm{Ar} /{ }^{39} \mathrm{Ar}$ method (Turner, 1969). Stepwise heating of six L-chondrites with nominal K-Ar ages from 1.0 to 1.85 aeons showed that the least retentive ${ }^{40} \mathrm{Ar}$ sites in each meteorite had been completely outgassed in a single event $500 \pm 30$ million yr ago; the higher ages for the bulk meteorites represent incomplete outgassing of the more retentive sites. Two other L-chondrites gave shorter ages $(305 \pm 30$ million yr), implying partial or complete outgassing at a later time.'

The size of the chondrite parent bodies can be estimated again from cooling rates, as for the irons. Wood (1967) has shown that most ordinary chondrites have cooled through $500 \mathrm{~K}$ at rates between 2 and $10 \mathrm{~K} /$ million yr. These limits correspond to depths of 40 to $150 \mathrm{~km}$ in bodies of $R \geqslant 150 \mathrm{~km}$ and 20 to $80 \mathrm{~km}$ in bodies of $R \geqslant 90 \mathrm{~km}$. Similar cooling rates, 5 to $9 \mathrm{~K} /$ million yr, have been estimated from ${ }^{129} \mathrm{Xe}$ diffusion (Manuel et al., 1968). They are also supported by various estimates of cooling times. (See Anders, 1971, for discussion and references.) It would seem that the parent bodies of ordinary chondrites were of about the same size as those of the irons.

There are a few skeletons in this closet, however. Unequilibrated chondrites of three chemical groups and type III carbonaceous chondrites gave lower 
cooling rates, 0.2 to $1 \mathrm{~K} /$ million $\mathrm{yr}$, corresponding to depths of 70 to $150 \mathrm{~km}$ in bodies $\geqslant 400 \mathrm{~km}$ in radius. This is about the size of Ceres; and though one cannot rule out the possibility that another Ceres-sized asteroid once existed but was destroyed, it does not seem very plausible that this one body should be the source of the least recrystallized, most primitive meteorites, and from four chemically distinct classes at that. Perhaps the metallographic method becomes unreliable in systems containing stony phases in addition to metal. Exceedingly low cooling rates were obtained for two other silicate-containing classes: pallasites, $0.4 \mathrm{~K} /$ million $\mathrm{yr}$, and mesosiderites, $0.1 \mathrm{~K} /$ million $\mathrm{yr}$. The latter corresponds to a temperature drop of only $450 \mathrm{~K}$ during the entire age of the solar system.

\section{POSSIBLE CANDIDATES}

\section{Comets}

Comets are too numerous and too small to serve as the principal source of meteorites. Öpik (1965) has estimated the number of extinct, short-period comets with aphelion distance smaller than $4.94 \mathrm{AU}$ as $2 \times 10^{4}$ to $10^{5}$. A significant fraction of these must have perihelia less than $1 \mathrm{AU}$. Even if one assumes that only a special subset of this population (e.g., low-velocity objects) can contribute meteorites, the resulting number far exceeds the apparent number of meteorite parent bodies, about 6 to 10. Available estimates of comet sizes (Roemer, 1971; Whipple, 1963) show them to be one to two orders of magnitude smaller than the meteorite parent bodies. Larger comets undoubtedly exist, but it is difficult to see how one giant comet, disrupted 520 million yr ago, could furnish half of Earth's meteorite influx. It is also hard to reconcile the fragility and high volatile content of comets with the prolonged high-temperature history of meteorite parent bodies and with the texture, chemistry, and mechanical strength of meteorites.

\section{Asteroids}

At first sight, the small number of meteorite parent bodies would seem to be incompatible with an asteroidal origin because asteroids, too, are very numerous. More than 4000 are known and at least 10 times as many undiscovered ones are thought to exist in the telescopically observable size range alone. However, two factors very greatly reduce this number. To change a typical asteroidal orbit into a meteoritic one, an acceleration of about $6 \mathrm{~km} / \mathrm{s}$ is required. Cratering theory and experiments show that only a minute fraction of the ejecta in a hypervelocity impact can be accelerated to this velocity, and because half the total energy appears as heat, such material will be vaporized. The absence of lunar basalts from Earth's meteorite collections shows that acceleration of rocks to $\geqslant 2.4 \mathrm{~km} / \mathrm{s}$ is indeed a very improbable process. Thus the majority of asteroids cannot contribute to Earth's meteorite influx. 
One group of asteroids that does not fall under this limitation is the Mars-crossing asteroids (Anders, 1964). Traversing the main part of the asteroid belt during every revolution, they suffer impacts from time to time. The debris, being ejected with low velocities, will move in similar, Mars-crossing orbits, but with slightly different periods. Consequently it soon spreads out in a toroid along the orbit of the parent body. Secular perturbations further disperse the fragments; and in a steady state, some of them are always in orbits intersecting the orbit of Mars. Close encounters with Mars reorient the velocity vectors of the debris (Arnold, 1965; Öpik, 1951), leading to Earth-crossing orbits in a fraction of cases. Thus Mars-crossing asteroids can serve as a source of meteorites.

The number of Mars asteroids, 34 according to the 1964 Ephemeris volume, is somewhat larger than the apparent number of meteorite parent bodies. However, 21 of these, comprising 98 percent of the mass and 92 percent of the cross section, belong to four Hirayama families (Anders, 1964). Thus the number of original Mars asteroids is indeed of the same order as the number of meteorite parent bodies.

Let us see how the picture changes when we make more optimistic assumptions about the escape of meteorites from the asteroid belt. Some asteroids of high inclination that are not Mars crossing at present will periodically become so, owing to secular perturbations (e.g., Pallas; Smith, 1964). Data are not available for most asteroids of interest, and would, moreover, not be quite appropriate for their debris, which moves in slightly different orbits. To obtain some sort of upper limit on the number of asteroids that can contribute meteorites, let us calculate $U$, the velocity relative to a hypothetical circular orbit at the same semimajor axis $a$ (Öpik, 1951):

$$
U^{2}=2\left(1-\sqrt{1-e^{2}} \cos i\right)
$$

The components of $\mathbf{U}$ in the $x, y$, and $z$ directions are

$$
\begin{aligned}
& U_{x}=e \\
& U_{z}=\sqrt{1-e^{2}} \sin i \\
& U_{y}=\sqrt{1-e^{2}} \cos i-1=\text { const }
\end{aligned}
$$

Minimum perihelion $q_{\min }$ is reached when $\mathrm{U}$ is reoriented such that $U_{z}$ and $i=0$, causing $e$ to reach $e_{\max }$. At each value of $a$, only debris from asteroids with $U$ greater than some minimum value has a chance of reaching the orbit of Mars, and then only in the doubly favorable case that perturbations cause $U_{z}$ to approach zero and cause the node to occur at or near minimum perihelion.

Whether such reorientation can actually take place is completely uncertain. One must appeal to unknown or ill-understood effects; e.g., higher order terms in secular perturbations or commensurabilities with Jupiter. However, it may be significant that the asteroids with highest eccentricities $(719,887,1036$, 
6344) occur near the $1 / 3$ commensurability, $a=2.50 \mathrm{AU}$. Perhaps a substantial reorientation of $\mathbf{U}$ takes place when $a$ is close to a major commensurability. Because all Mars-crossing asteroid families have $a$ within $0.2 \mathrm{AU}$ of the $1 / 3$, $1 / 4$, or $2 / 5$ commensurabilities, some fraction of their ejecta will have commensurable orbits. They will certainly be subject to strong Jupiter perturbations, perhaps of the required kind.

[Note added after colloquium: There now exists some support for these speculations. Williams ${ }^{1}$ has found, using his new theory of secular perturbations, that several resonance surfaces exist in $a, e^{\prime}, \sin i^{\prime}$ space in the inner asteroid belt. Any object in the vicinity of these resonances will experience very large oscillations in $e$ and $i$, which of course favor attainment of a Mars-crossing orbit. Interestingly, each of the high-velocity asteroid families in figure 1 adjoins one or more of these resonance surfaces. A significant fraction of their collisional debris thus will be thrown into these resonance regions, where the postulated reorientation of $\mathbf{U}$ may take place. Williams' resonances may thus be the long-sought factor permitting change of highly inclined to highly eccentric orbits.]

The distribution of $U$ is illustrated in figure 1 for the inner half of the asteroid belt. The minimum value of $U$ required to reach $q=1.700 \mathrm{AU}$ (a value part way between the present and maximum aphelion of Mars) is indicated by the solid line. Asteroids lying above the line are potential sources of Mars-crossing debris under the above, optimistic assumptions.

Nearly one-tenth of all asteroids have $q_{\text {min }}$ less than $1.700 \mathrm{AU}$. Yet the number of potential meteorite parent bodies has not increased greatly. Many of the newly added asteroids are less eccentric but otherwise bona fide members of Mars-crossing families 5, 29, 30, and $31 .^{2}$ Others fall within the boundaries of these families on a $U$ versus $a$ plot, but have $e^{\prime}$ and $i^{\prime}$ outside the family limits. They are either interlopers or former members whose elements were changed by Mars encounters.

Only three new families appear on this graph. The cluster at $1.9 \mathrm{AU}$, though quite disperse on an $a, e^{\prime}, i^{\prime}$ plot, is fairly compact on a $U, a$ plot. Nine additional members of this cluster were discovered by van Houten et al. (1970), who referred to it as the "Hungaria group." Family 28, including 2 Pallas, becomes marginally Mars crossing every $10^{4}$ yr (Smith, 1964) but its velocity is high enough to give a potential $q_{\min }$ as small as $1.10 \mathrm{AU}$ if $i=0$. In fact, family 30 may have been derived from family 28 by a partial reorientation of U. Family 17 is a very marginal case, but has been included for the sake of completeness.

\footnotetext{
${ }^{1}$ See p. 177.

${ }^{2}$ The status of family 31 is in some jeopardy. Anders (1965) suggested that it might be related to the Flora families, 6 to 9. Arnold (1969) has questioned its reality on the grounds that it constitutes only a twofold enhancement of asteroid density in $a, e^{\prime}, i^{t}$ space over the general "background." Van Houten et al. (1970) have assigned number 31 to another family, not being aware of the previous assignment.
} 


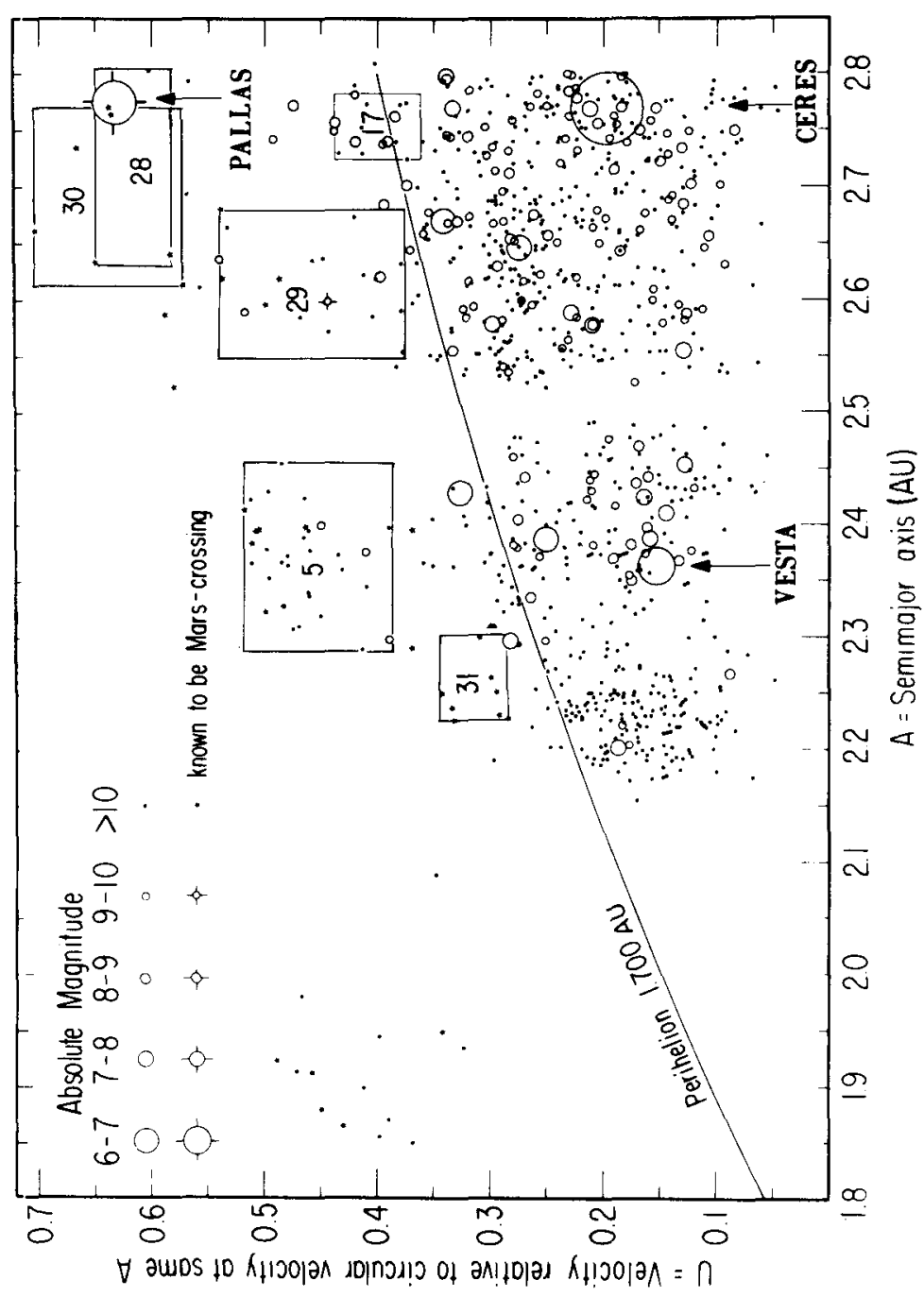

壹造

震

焉

焉

焉

ญ

is

$\infty$ :

Is

运

응

焉

र

引

녕을

음

窝若:

흥하년

응 해을

$\sum$.

o

을

5

를

के

온

동

है

ชี่

焉

5

웧

롤

岕范

过

홍

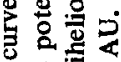

올

的䒕

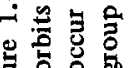
运 
Only a few nonfamily asteroids fall above the $q=1.700 \mathrm{AU}$ line, most of them by very marginal amounts. The only sizable objects are 18 Melpomene at $2.296 \mathrm{AU}$ (possibly related to family 31 ), 6 Hebe at $2.426 \mathrm{AU}, 247$ Eukrate at $2.741 \mathrm{AU}$, and $148 \mathrm{Gallia}$ at $2.771 \mathrm{AU}$. A list of actual or potential Mars crossers is given in table III. Original radii were reconstructed according to Anders (1965), using data for the first 1651 numbered asteroids from the 1964 Ephemeris volume. A geometric albedo of 0.12 , as for Ceres, was assumed, resulting in the magnitude-radius relationship

$$
\log R=3.385-0.200 g
$$

We see that the reconstructed family asteroids (table III) are only about one-half as large or one-eighth as massive as the meteorite parent bodies (table I). This is not surprising, because the family asteroids now visible represent but a fraction of the original population. If we ascribe the discrepancy to loss of $7 / 8$ of the members by dispersal or deflection to terrestrial space, the combined half-life for these processes must be on the order of 1 to 2 aeons, depending on the age of the family. This is roughly consistent with the half-lives found in Monte Carlo calculations, and with Dohnanyi's (1969) estimated lifetimes for collisional destruction of asteroids 10 to $20 \mathrm{~km}$ in radius. The half-lives may be longer if the albedo of the asteroids is smaller than assumed. A value of 0.065 , as for Phobos (Smith, 1970), would reduce the mean discrepancy in mass to a factor of $\sim 3$ and would lengthen the half-life accordingly.

We note in passing that the two most massive objects in table III, 2 Pallas and 6 Hebe, are not extensively broken up, and hence probably cannot serve as

\section{TABLE III.-Actual and Potential Mars-Crossing Asteroids}

\begin{tabular}{|c|c|c|c|c|c|c|c|}
\hline Family & $\begin{array}{c}\text { Number } \\
\text { of } \\
\text { members }\end{array}$ & $\begin{array}{l}\text { Representative } \\
\text { member }\end{array}$ & $\begin{array}{c}a, \\
\mathrm{AU}\end{array}$ & $\begin{array}{l}q \\
\mathrm{AU}\end{array}$ & $q_{\min }{ }^{a}$ & $U$ & $\begin{array}{l}R_{0} \\
\mathrm{~km}\end{array}$ \\
\hline Hungaria & 23 & 1235 Schorria & 1.910 & 1.617 & 1.060 & 0.457 & $\mathrm{~b}_{11}$ \\
\hline 31 & 9 & 1204 Renzia & 2.264 & 1.596 & 1.592 & .300 & $c_{71}$ \\
\hline 5 Phocaea & 34 & 1310 Villigera & 2.392 & 1.538 & 1.219 & .507 & $b_{53}$ \\
\hline 30 Aethra & 4 & 1036 Ganymed & 2.658 & 1.216 & .910 & .703 & $b_{28}$ \\
\hline 29 Desiderata & 7 & 1134 Kepler & 2.683 & 1.432 & 1.289 & .539 & $b_{53}$ \\
\hline 17 & 11 & 36 Atalante & 2.749 & 1.924 & 1.576 & .437 & $b_{85}$ \\
\hline 28 Pallas & 6 & 2 Pallas & 2.772 & 2.123 & 1.103 & .635 & 245 \\
\hline Hebe & 1 & 6 Hebe & 2.426 & 1.934 & 1.646 & .326 & 116 \\
\hline
\end{tabular}

\footnotetext{
${ }^{a}$ For zero inclination.

$\mathrm{b}_{\text {Highly fragmented. }}$

${ }^{c}$ Including 18 Melpomene; without this asteroid, $R_{0}$ would be $5 \mathrm{~km}$ and the classification changed to highly fragmented.
} 
sources of iron meteorites. Most or all irons in each group apparently came from the deep interiors of their parent bodies (Fricker et al., 1970). But if the dispersal-deflection half-life is as short as 1 to 2 aeons, some older families may have been decimated, fragmented, and dispersed beyond recognition. Their remains are presumably hidden in the nonfamily background in figure 1 .

Thus far we have relied entirely on planetary perturbations to extract meteorites from the asteroid belt, neglecting the effect of ejection velocity. Actually, it appears that ejection velocity was an important factor in at least one case, the group III irons. Jaeger and Lipschutz (1967) have noted that group III irons, without exception, are shocked to $>13 \mathrm{GN} / \mathrm{m}^{2}(130 \mathrm{~kb})$, often to $>75 \mathrm{GN} / \mathrm{m}^{2}(750 \mathrm{~kb})$. Shock pressures of this order correspond to free-surface velocities of 1 to $3 \mathrm{~km} / \mathrm{s}$; and the fact that no lightly shocked members are found in group III (in contrast to other groups) suggests that high shock pressures, and the concomitant acceleration, were essential to the escape of these meteorites from the asteroid belt. Jaeger and Lipschutz propose that the parent body of group III was a ring asteroid not crossing the orbit of Mars. Only its high-velocity ejecta had a chance of achieving a Mars-crossing orbit, the essential prerequisite for deflection into terrestrial space.

Various objections have been raised to Mars asteroids as the principal source of meteorites. Öpik $(1965,1968)$ has pointed out that their mean lives for deflection into Earth-crossing orbits, $10^{9}$ to $10^{10} \mathrm{yr}$, are far longer than the radiation ages of stony meteorites, $2 \times 10^{4}$ to $6 \times 10^{7} \mathrm{yr}$. He maintains that such short capture times are completely unattainable for debris from Mars asteroids. However, it is important to make a distinction between mean capture times for a large population and actual capture times for individual objects. On the Mars asteroid model, the radiation age $T$ is the sum of two intervals: $t_{1}$, from ejection of the meteorite from its parent body to deflection into an Earth-crossing orbit, and $t_{2}$, from achievement of the first Earth-crossing orbit to actual capture by Earth. Both $t_{1}$ and $t_{2}$ are exponentially distributed about the mean lives for the two processes, $\tau_{1}$ and $\tau_{2}$, and, because the most probable values of $t_{1}$ and $t_{2}$ in an exponential distribution are zero, small values of $T$ are not at all inconsistent with large values of $\tau_{1}$ and $\tau_{2}$. One can also prove this by recognizing that the toroidal debris stream associated with each Mars asteroid is analogous to a meteor stream. Typically, orbits of planet and stream intersect for a few centuries during each $10^{4} \mathrm{yr}$ oscillation in $e$ and $i$ If the stream is continuous, some objects will be captured or deflected during each revolution as the planet crosses the stream. Because the distribution of radiation ages along the stream is random, the objects deflected will include some very young ones.

Arnold (1965) and Wetherill (1967, 1968a) objected to a Mars asteroid origin mainly on the grounds that it would give a preponderance of long ages, in the range $10^{8}$ to $10^{9} \mathrm{yr}$. In principle, such long ages could be suppressed by collisional destruction of meteorites, but a careful analysis of the problem seemed to show that the density of dust and rubble in the asteroid belt was too 
low to give a short enough lifetime against collisional destruction; e.g., $10^{7} \mathrm{yr}$. However, there is good reason to believe that the destruction lifetime is indeed on this order. Dohnanyi (1969) has reexamined the problem using an improved mass distribution function and cratering relations. He obtains mean destruction lifetimes of 3 to 10 million $y r$ for objects 10 to $100 \mathrm{~cm}$ in diameter. Mazor et al. (1970) and Herzog (1970) have pointed out the curious fact that the radiation age distributions of meteorites show cutoffs related to crushing strength: $\sim 15$ million yr for the friable carbonaceous chondrites, $\sim 60$ million $\mathrm{yr}$ for all other stones, $\sim 200$ million yr for stony irons, and $\sim 2$ billion yr for irons. It appears that the age distribution is indeed governed by collisional destruction. Cutoffs of exactly the right order have been produced in Monte Carlo calculations, using a destruction lifetime of 10 million yr (Mellick and Anders, unpublished).

Of course, if the majority of stony meteorites are destroyed by collisions, a correspondingly more intense source is needed to maintain the meteorite flux observed on Earth $\left(10^{5}\right.$ to $\left.10^{6} \mathrm{~kg} / \mathrm{yr}\right)$. The potential reservoir, from figure 1, is probably on the order of $10^{19}$ to $10^{20} \mathrm{~kg}$, so that even an extraction efficiency as low as $10^{-2}$ to $10^{-3}$ would suffice to maintain this flux for $10^{9}$ to $10^{10} \mathrm{yr}$. However, if the correlations of $\omega$ (the argument of perihelion) and $e$ noted by Wetherill (1968a) prevent node and perihelion from coinciding even over periods of $>10^{7} \mathrm{yr}$, and even for ejecta, then there may indeed be a problem.

Another observation to be explained is the predominance of p.m. falls among the chondrites. Wetherill $(1968 b, 1969)$ has pointed out that this requires a large orbit of low inclination. Moreover, the meteorite must be captured by Earth during the first few passes, otherwise $a$ decreases, $i$ increases, and the a.m./p.m. asymmetry is lost. Wetherill suggested that a special class of low-velocity, short-period, cometary orbits with aphelia near Jupiter would be suitable because objects in such orbits, if not quickly captured by Earth, are soon eliminated by Jupiter. However, it is difficult to envision circumstances where this type of orbit would dominate over more conventional short-period cometary orbits with higher geocentric velocities and/or smaller aphelia. It seems that the a.m./p.m. asymmetry can be equally well explained by the asteroidal model if collisional destruction is invoked to prevent "evolution" of the orbit by repeated close encounters with Earth. With a destruction lifetime of 3 to 10 million yr (Dohnanyi, 1969), meteorites will be captured by Earth in the first few passes, if at all.

An unsolved problem still remaining is the relatively high frequency of meteorites with high geocentric velocities, $U_{e}=0.5$ to 0.7 . Data are limited and of variable quality, but it appears from the available information on photographic and visual meteorite orbits (Millman, 1969), dense ("asteroidal") meteors, and Apollo asteroids that perhaps one-third of all meteorites have velocities in this range. Monte Carlo calculations for all Mars asteroid families give such velocities in much lower abundance (Anders and Mellick, 1969; 
Mellick and Anders, unpublished). In principle, the required acceleration could be achieved by an appulse to Jupiter, but such appulses lead to crossings and subsequent rapid elimination of the object. Perhaps commensurabilities or other factors stabilize some types of large orbit long enough for Earth capture to compete with Jupiter ejection. A theoretical investigation of this problem would be very desirable.

\section{COMETARY CONTRIBUTION TO THE METEORITE FLUX}

If most meteorites come from Mars asteroids, where then is the cometary debris? Three major clues are available: meteors, Apollo asteroids, and meteoritic material on the Moon.

\section{Meteors}

It appears that the majority of photographic meteors, including the Prairie Network fireballs, are of cometary origin $\left(\mathrm{McCrosky}^{3}\right)$. An asteroidal component seems to be present (Ceplecha, 1966; Kresák, 1969a,b) but is clearly subordinate in this mass range.

\section{Apollo Asteroids}

The Apollo asteroids seem to fall into two groups differing in geocentric velocity (table IV). Anders and Arnold (1965) suggested that the low-velocity group was asteroidal and the high-velocity group, cometary. Some support for this division has been obtained by Gehrels and his associates. The "cometary"

TABLE IV.-Apollo Asteroids

[Wetherill and Williams, 1969]

\begin{tabular}{|c|c|c|c|}
\hline Asteroid & $\begin{array}{c}a \\
\mathrm{AU}\end{array}$ & $\mathrm{U}_{e}$ & $\begin{array}{l}R, \\
\mathrm{~km}\end{array}$ \\
\hline 1620 Geographos $^{\mathrm{a}}$ & 1.244 & 0.393 & 1.62 \\
\hline 1685 Toro & 1.382 & .443 & 2.82 \\
\hline $1950 \mathrm{DA}$ & 1.695 & .451 & 1.02 \\
\hline Hermes & 1.29 & .481 & .39 \\
\hline $1948 \mathrm{EA}$ & 2.261 & .536 & 2.95 \\
\hline Apollo & 1.48 & .569 & .39 \\
\hline $1959 \mathrm{LM}$ & 2.155 & .624 & 2.45 \\
\hline Adonis & 1.97 & .852 & .15 \\
\hline 1580 Betulia & 2.195 & .978 & 1.86 \\
\hline 1566 Icarus $^{\mathrm{b}}$ & 1.078 & .995 & .54 \\
\hline Comet Encke & 2.22 & 1.002 & \\
\hline
\end{tabular}

${ }^{\text {a }}$ Axial ratio is 3.4 (Gehrels et al., 1970).

${ }^{\mathrm{b}}$ Axial ratio is 1.1 (Gehrels et al., 1970).

${ }^{3}$ See p. 395. 
object 1566 Icarus shows only a minor variation $(\leqslant 0.24 \mathrm{mag})$ in its lightcurve, implying a nearly spherical shape, to $\leqslant 10$ percent (Gehrels et al., 1970). According to Dohnanyi (1969), an object $0.54 \mathrm{~km}$ in radius, situated in the asteroid belt, has a lifetime of only $2.5 \times 10^{8} \mathrm{yr}$ against collisional destruction. It seems inconceivable that an object as small as Icarus could have maintained its spherical shape for 4.5 aeons if it had resided in the asteroid belt during that time. Oort's comet belt, on the other hand, would be an acceptable, relatively collision-free place of storage.

The asteroidal object 1620 Geographos, on the other hand, has a strongly elongated shape, with axial ratio 3.4:1 (Gehrels et al., 1970). This is entirely reasonable for an object that spent its entire life in the asteroid belt.

If geocentric velocity is accepted as the criterion, three of the objects in table IV are cometary and seven asteroidal. (Comet Encke must be omitted because its discovery was aided by its light emission.) On a mass basis, the cometary contribution would seem to be smaller than 30 percent, but in view of the limited statistics, not much can be made of this trend.

\section{Moon}

A number of trace elements ( $\mathrm{Au}, \mathrm{Ir}, \mathrm{Bi}, \mathrm{Te}$, etc.) are enriched in Apollo 11 lunar soils and breccias relative to igneous rocks, apparently reflecting addition of a meteoritic component (Ganapathy et al., 1970). From the abundance pattern it appears that this component consists largely of primitive, "carbonaceous-chondrite-like" material. The amount is about 1.9 percent, corresponding to an average influx rate of $4 \times 10^{-9} \mathrm{~g}-\mathrm{cm}^{-2}-\mathrm{yr}^{-1}$. This agrees within a factor of 3 with a similar estimate for Earth, based on the Ir and Os content of Pacific and Indian Ocean sediments (Barker and Anders, 1968).

Apollo 12 soils collected some distance away from craters showed a very similar pattern, whereas those collected on crater rims gave a different pattern, resembling fractionated meteorites (irons, ordinary chondrites) in their low abundance of $\mathrm{Bi}$, for example (Laul et al., 1971).

Six different impacts have thus far been characterized, and it seems that five of them were caused by fractionated meteorites (table V). On the basis of these

TABLE V.-Meteorite Impacts on Moon

[Laul et al., 1971]

\begin{tabular}{l|c|l}
\hline \multicolumn{1}{c|}{ Event } & $\begin{array}{c}\text { Crater } \\
\text { diameter, } \mathrm{km}\end{array}$ & $\begin{array}{l}\text { Projectile } \\
\text { composition }\end{array}$ \\
\hline 12017 Glass & $?$ & Primitive \\
Bench Crater & .07 & Fractionated \\
Head Crater & .13 & Fractionated \\
Surveyor Crater & .18 & Fractionated \\
10085 Anorthosite & $?$ & Fractionated \\
Copernicus? & 91 & Fractionated \\
\hline
\end{tabular}


limited statistics, it appears that fractionated material dominates among the larger $(>1 \mathrm{~kg})$ bodies falling on both Earth and the Moon. Primitive material, on the other hand, seems to dominate among the subkilogram objects that are apparently responsible for the uniform blanket of $\mathrm{C} 1$-like material covering the Moon and for fireballs or meteors on Earth.

Probably the primitive component consists mainly of the debris of spontaneously disintegrating comets, with an unknown asteroidal contribution. Comets are rich in volatiles and hence almost certainly are of primitive composition. However, any material accreted at temperatures below $\sim 400 \mathrm{~K}$ is likely to have this composition. An additional source of such material may thus be asteroids from the outer part of the belt and the surface layers of all asteroids.

\section{ORIGIN OF METEORITES}

Great efforts have been made to understand the chemical and thermal history of meteorites, starting with Urey's $(1952,1954)$ classic papers. It appears that the observed chemical fractionations, involving some 55 elements, are due to only four basic processes that occurred in the solar nebula during cooling from high temperatures. I have reviewed the subject in a recent paper (Anders, 1971) and will therefore merely summarize the model that best accounts for the evidence (fig. 2). Degree of condensation is plotted on the ordinate; and degree of retention, on the abscissa.

(1) An early condensate, containing refractory elements ( $\mathrm{Ca}, \mathrm{Al}, \mathrm{Ti}, \mathrm{U}$, $\mathrm{Th}$, lanthanides, Pt metals, etc.) was partially lost from ordinary and enstatite chondrites.

(2) After condensation of the remaining material to grains of $10^{-5}$ to $10^{-6} \mathrm{~cm}$, some of the nickel-iron was lost, at a temperature around $700 \mathrm{~K}$. During this and the following stages, the enstatite chondrites apparently found themselves in a more reducing environment, perhaps a gas phase of $\mathrm{C} / \mathrm{O} \geqslant 0.9$.

(3) During or after the metal loss, about 30 to 80 percent of the condensate was remelted to millimeter-sized droplets by local heating events on a time scale of seconds to minutes (probably electric discharges; Whipple, 1966). Volatiles were lost from the remelted material.

(4) The unremelted, fine-grained material continued to take up volatiles from the nebula $(\mathrm{Pb}, \mathrm{Bi}, \mathrm{Tl}, \mathrm{In}$, etc.) and accreted with the remelted material. Accretion took place at $P=10^{-4 \pm 2}$ atm and falling temperatures, as indicated in figure 2 . The values for carbonaceous and enstatite chondrites are only rough estimates.

The five principal chondrite classes were affected by these processes to a markedly different extent. Carbonaceous chondrites were generally affected least, and enstatite chondrites, most. Presumably this reflects differences in place and time of formation. If we only knew the original location of their 


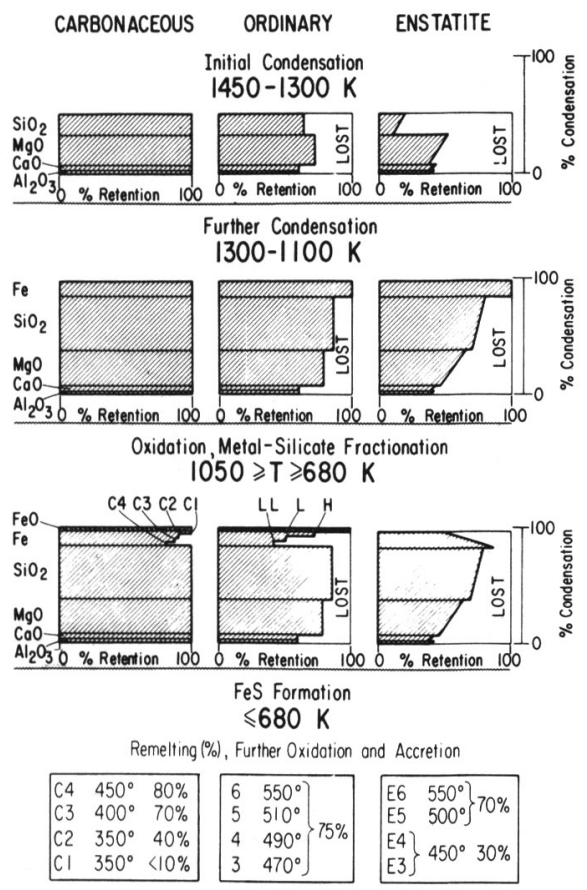

Figure 2.-Proposed outline of chondrite formation in a cooling solar nebula (Larimer and Anders, 1970). Shaded areas represent condensed phase at end of each stage. Fraction condensed is shown on ordinate; amount remaining after fractionation, on abscissa. Four partial fractionations seem to have taken place: (1) loss of initial condensate at $\sim 1300 \mathrm{~K}$, (2) loss of metal at $\sim 700 \mathrm{~K}$, (3) remelting at $\sim 500$ to $600 \mathrm{~K}$, and (4) accretion at 350 to $550 \mathrm{~K}$. The three main chondrite classes presumably represent three to five different regions of the inner solar nebula. Boxes at bottom of figure give estimated accretion temperatures of each petrologic type and percent remelted, volatile-poor materials. Numbers refer to petrologic types within each chondritic class.

parent bodies, we could correlate all this information with heliocentric distance, and thus reconstruct the chemical and thermal history of the inner solar nebula in considerable detail.

\section{ACKNOWLEDGMENTS}

I am indebted to Michael S. Lancet and Rudy Banovich for the preparation of figure 1. This work was supported in part by the U.S. AEC Contract AT(11-1)-382 and NASA Grant NGL 14-001-010.

\section{REFERENCES}

Anders, E. 1964, Origin, Age, and Composition of Meteorites. Space Sci. Rev. 3, 583-714. Anders, E. 1965, Fragmentation History of Asteroids. Icarus 4, 399-408.

Anders, E. 1971, Meteorites and the Early Solar System. Ann. Rev. Astron. Astrophys. 9, $1-34$. 
Anders, E., and Arnold, J. R. 1965, Age of Craters on Mars. Science 149, 1494-1496.

Anders, E., and Mellick, P. J. 1969, Orbital Clues to the Nature of Meteorite Parent Bodies. Meteorite Research (ed., P. M. Millman), ch. 47, pp. 560-572. D. Reidel. Dordrecht.

Amold, J. R. 1965, The Origin of Meteorites as Small Bodies. II. The Model. Astrophys. J. 141, 1536-1547.

Arnold, J. R. 1969, Asteroid Families and "Jet Streams." Astron. J. 74, 1235-1242.

Barker, J. L., Jr., and Anders, E. 1968, Accretion Rate of Cosmic Matter From Iridium and Osmium Contents of Deep-Sea Sediments. Geochim. Cosmochim. Acta 32, 627-745.

Carter, N. L., Raleigh, C. B., and DeCarli, P. S. 1968, Deformation of Olivine in Stony Meteorites. J. Geophys. Res. 73, 5439-5461.

Ceplecha, Z. 1966, Classification of Meteor Orbits. Bull. Astron. Inst. Czech. 17, 96-98.

Christophe, Michel-Lévy, M. C. 1969, Comparaison de Certains Aspects de la Structure Microscopique des Chondrites Avec Leur Âge Apparent de Rétention Gazeuse. C. R. Acad. Sci. Paris 268, 2853-2855.

Dohnanyi, J. S. 1969, Collisional Model of Asteroids and Their Debris. J. Geophys. Res. 74, 2531-2554.

Fleischer, R. L., Price, P. B., and Walker, R. M. 1968, Identification of $\mathrm{Pu}^{244}$ Fission Tracks and the Cooling of the Parent Body of the Toluca Meteorite. Geochim. Cosmochim. Acta 32, 21-31.

Fricker, P. E., Goldstein, J. I., and Summers, A. L. 1970, Cooling Rates and Thermal Histories of Iron and Stony-Iron Meteorites. Geochim. Cosmochim. Acta 34, 475-491.

Ganapathy, R., Keays, R. R., Laul, J. C., and Anders, E. 1970, Trace Elements in Apollo 11 Lunar Rocks: Implications for Meteorite Influx and Origin of Moon. Proc. Apollo 11 Lunar Sci. Conf. Geochim. Cosmochim. Acta 34, suppl. 1, 1117-1142.

Gehrels, T., Roemer, E., Taylor, R. C., and Zellner, B. H. 1970, Minor Planets and Related Objects. IV. Asteroid (1566) Icarus. Astron. J. 75, 186-195.

Goldstein, J. I., and Doan, A. S., Jr. 1971, The Effect of Phosphorus on the Formation of the Widmanstätten Pattern in Iron Meteorites. Geochim. Cosmochim. Acta 35, in press.

Goldstein, J. I., and Ogilvie, R. E. 1965, The Growth of the Widmanstätten Pattern in Metallic Meteorites. Geochim. Cosmochim. Acta 29, 893-920.

Goldstein, J. I., and Short, J. M. 1967a, The Iron Meteorites, Their Thermal History, and Parent Bodies. Geochim. Cosmochim. Acta 31, 1733-1770.

Goldstein, J. I., and Short, J. M. 1967b, Cooling Rates of 27 Iron and Stony-Iron Meteorites. Geochim. Cosmochim. Acta 31, 1001-1023.

Herzog, G. F. 1970, A Revised Radiation Age for Norton County Meteorite. Paper presented at National Fall Meeting, Amer. Geophys. Union (San Francisco).

Heymann, D. 1967, On the Origin of Hypersthene Chondrites: Ages and Shock-Effects of Black Chondrites. Icarus 6, 189-221.

Houten, C. J. van, Houten-Groeneveld, I. van, Herget, P., and Gehrels, T. 1970, Palomar-Leiden Survey of Faint Minor Planets. Astron. Astrophys. Suppl. Ser. 2, 339-448.

Jaeger, R. R., and Lipschutz, M. E. 1967, Implications of Shock Effects in Iron Meteorites. Geochim. Cosmochim. Acta 31, 1811-1832.

Kresák, L. 1969a, The Discrimination Between Cometary and Asteroidal Meteors-I. The Orbital Criteria. Bull. Astron. Inst. Czech. 20, 177-188.

Kresák, L. 1969b, The Discrimination Between Cometary and Asteroidal Meteors-II. The Orbits and Physical Characteristics of Meteors. Bull. Astron. Inst. Czech. 20, 232-251.

Larimer, J. W., and Anders, E. 1970, Chemical Fractionations in Meteorites-III. Major Element Fractionations in Chondrites. Geochim. Cosmochim. Acta 34, 367-388.

Laul, J. C., Morgan, J. W., Ganapathy, R., and Anders, E. 1971, Meteoritic Material in Lunar Samples: Characterization From Trace Elements. Proc. Apollo 12 Lunar Sci. Conf. Geochim. Cosmochim. Acta 35, suppl. 1. 
Manuel, O. K., Alexander, E. C., Jr., Roach, D. V., and Ganapathy, R. $1968,{ }^{129} \mathrm{I}^{129} \mathrm{Xe}$ Dating of Chondrites. Icarus 9, 291-304.

Mazor, E., Heymann, D., and Anders, E. 1970, Noble Gases in Carbonaceous Chondrites. Geochim. Cosmochim. Acta 34, 781-824.

Millman, P. M. 1969, Astronomical Information on Meteorite Orbits. Meteorite Research (ed., P. M. Millman), ch. 45, pp. 541-551. D. Reidel. Dordrecht.

Öpik, E. J. 1951, Collision Probabilities With the Planets and Distribution of Interplanetary Matter. Proc. Roy. Irish Acad. Sect. A 54, 165-199.

Öpik, E. J. 1965, The Stray Bodies in the Solar System. Part II. The Cometary Origin of Meteorites. Adv. Astron. Astrophys. 4, 301-336.

Öpik, E. J. 1968, The Cometary Origin of Meteorites. Irish Astron. J. 8, 185-208.

Roemer, E. 1971, Long-Focus Observations. Paper presented at 3d Ann. Meeting, Div. Planet. Sci., Amer. Astron. Soc. (Tallahassee).

Smith, A. J., Jr. 1964, A Discussion of Halphen's Method for Secular Perturbations and Its Application to the Determination of Long Range Effects in the Motions of Celestial Bodies. Pt. 2. NASA TR R-194.

Smith, B. A. 1970, Phobos: Preliminary Results From Mariner 7. Science 168, 828-830.

Taylor, G. J., and Heymann, D. 1969, Shock, Reheating, and the Gas Retention Ages of Chondrites. Earth Planet. Sci. Lett. 7, 151-161.

Turner, G. 1969, Thermal Histories of Meteorites by the ${ }^{39} \mathrm{Ar}^{-40} \mathrm{Ar}$ Method. Meteorite Research (ed., P. M. Millman), ch. 34, pp. 407-417. D. Reidel. Dordrecht.

Urey, H. C. 1952, Chemical Fractionation in the Meteorites and the Abundance of the Elements. Geochim. Cosmochim. Acta 2, 269-282.

Urey, H. C. 1954, On the Dissipation of Gas and Volatilized Elements From Protoplanets. Astrophys. J. Suppl. Ser. 1(6), 147-173.

Urey, H. C. 1966, Chemical Evidence Relative to the Origin of the Solar System. Mon. Notic. Roy. Astron. Soc. 131, 199-223.

Van Schmus, W. R. 1969, The Mineralogy and Petrology of Chondritic Meteorites. Earth Sci. Rev. 5, 145-184.

Van Schmus, W. R., and Ribbe, P. H. 1968, The Composition and Structural State of Feldspar From Chondritic Meteorites. Geochim. Cosmochim. Acta 32, 1327-1342.

Voshage, H. 1967, Bestrahlungsalter und Herkunft der Eisenmeteorite. Z. Naturforsch. A 22, 477-506.

Wänke, H. 1966, Meteoritenalter und verwandte Probleme der Kosmochemie. Fortschr. Chem. Forsch. 7, 322-408.

Wasson, J. T. 1969, The Chemical Classification of Iron Meteorites-III. Hexahedrites and Other Irons With Germanium Concentrations Between 80 and $200 \mathrm{ppm}$. Geochim. Cosmochim. Acta 33, 859-876.

Wasson, J. T. 1970, The Chemical Classification of Iron Meteorites-IV. Irons With Ge Concentrations Greater Than $190 \mathrm{ppm}$ and Other Meteorites Associated With Group I. Icarus 12, 407-423.

Wetherill, G. W. 1967, Collisions in the Asteroid Belt. J. Geophys. Res. 72, 2429-2444.

Wetherill, G. W. 1968a, Dynamical Studies of Asteroidal and Cometary Orbits and Their Relation to the Origin of Meteorites. Origin and Distribution of the Elements (ed., L. H. Ahrens), pp. 423-443. Pergamon Press. Oxford.

Wetherill, G. W. 1968b, Time of Fall and Origin of Stone Meteorites. Science 159, 79-82. Wetherill, G. W. 1969, Relationships Between Orbits and Sources of Chondritic Meteorites. Meteorite Research (ed., P. M. Millman), ch. 48, pp. 573-589. D. Reidel Dordrecht.

Wetherill, G. W., and Williams, J. G. 1969, Evaluation of the Apollo Asteroids as Sources of Stone Meteorites. J. Geophys. Res. 73, 635-648.

Whipple, F. L. 1963, On the Structure of the Cometary Nucleus. The Moon, Meteorites, and Comets. Vol. IV of The Solar System (eds., B. M. Middlehurst and G. P. Kuiper), pp. 639-664. Univ. of Chicago Press. Chicago. 
Whipple, F. L. 1966, A Suggestion as to the Origin of Chondrules. Science 153, 54-56.

Wood, J. A. 1964, The Cooling Rates and Parent Planets of Several Iron Meteorites. Icarus $3,429-460$.

Wood, J. A. 1967, Chondrites: Their Metallic Minerals, Thermal Histories, and Parent Planets. Icarus 6, 1-49.

Wood, J. A. 1968, Meteorites and the Origin of Planets. McGraw-Hill Book Co., Inc. New York. 